ESJ Humanities

\title{
Caracterisation de l'espace frontalier du plateau au Sud-Est du Benin
}

\author{
Seidou Abdel Hack \\ Dekakon Satingo Rolette
}

Ecole Doctorale Pluridisciplinaire de

l'Université d'Abomey-Calavi, EDP-UAC, Benin

Zannou Sandé

Laboratoire de l'Aménagement du Territoire, de l'Environnement et de Développement Durable, LATEDD, Benin

Vigninou Toussaint

Laboratoire d'Etudes des Dynamiques Urbaines et Régionales (LEDUR),

Département de Géographie et Aménagement du Territoire (DGAT),

Université d'Abomey-Calavi, Benin

\section{Tente Brice}

Laboratoire de Biogéographie et Expertise Environnementale (LABEE)

Département de Géographie et Aménagement du Territoire (DGAT), Université d'Abomey-Calavi, UAC-Bénin

Doi:10.19044/esj.2022.v18n7p90

Submitted: 30 December 2021

Accepted: 24 February 2022

Published: 28 February 2022
Copyright 2022 Author(s)

Under Creative Commons BY-NC-ND

4.0 OPEN ACCESS

Cite As:

Abdel Hack S., Satingo Rolette D., Sandé Z., Toussaint V. \& Brice T. (2022). Caracterisation de l'espace frontalier du plateau au Sud-Est du Benin. European Scientific Journal, ESJ, 18 (7), 90. https://doi.org/10.19044/esj.2022.v18n7p90

\section{Résumé}

La présence d'une frontière favorise le développement des échanges commerciaux entre deux Etats. Ainsi, le commerce transfrontalier est une activité très développée dans les cinq Communes du Département du Plateau, un Département frontalier avec le Nigeria sur tout le coté Est. Les caractéristiques de l'espace frontalier du Plateau ont été étudiées par le présent travail. Pour l'atteinte des objectifs relatifs à la description des activités économiques et aux principaux produits objets d'échanges dans le secteur d'étude, des investigations ont été faites à partir de la collecte des données et informations aux moyens de la recherche documentaire, des observations directes sur le terrain, des entretiens et enquêtes auprès de 390 ménages 
d'acteurs du commerce transfrontalier ainsi que des interviews auprès de 51 personnes ressources et autorités du milieu d'étude. Les résultats obtenus ont été analysés et interprétés à l'aide du modèle FFOM (Forces, Faiblesses, Opportunités et Menaces). Les résultats obtenus révèlent que les marchés sont les principaux lieux qui structurent l'espace transfrontalier du Département du Plateau. Ce sont des lieux publics, fixes et sûrs de rencontre où acheteurs et vendeurs se bousculent voire rivalisent d'ardeur les jours d'animation. Cette activité est organisée à travers la transaction par voie fluviale de Kétoukpè jusqu'à hauteur de Banigbé dans la Commune d'Ifangni et par voie terrestre d'Igolo jusqu'à hauteur de Idigny dans la Commune de Kétou. Les produits échangés sont par ordre d'importance les produits de consommation alimentaire $(25,90 \%)$, les produits manufacturés non alimentaires $(22,89 \%)$, les produits pétroliers $(19,74 \%)$, les produits chimiques $(17,69 \%)$, les produits d'équipement $(9,23 \%)$ et les véhicules d'occasion $(4,62 \%)$. Enfin, cette activité a également des effets socio-économiques non négligeables.

Mots-clés : Département du Plateau, frontière, échanges, espace frontalier, produits 


\title{
Characterization of the Border Area of the Plateau in South-East Benin
}

\author{
Seidou Abdel Hack \\ Dekakon Satingo Rolette
}

Ecole Doctorale Pluridisciplinaire de

l'Université d'Abomey-Calavi, EDP-UAC, Benin

Zannou Sandé

Laboratoire de l'Aménagement du Territoire,

de l'Environnement et de Développement Durable, LATEDD, Benin

\section{Vigninou Toussaint}

Laboratoire d'Etudes des Dynamiques Urbaines et Régionales (LEDUR),

Département de Géographie et Aménagement du Territoire (DGAT),

Université d'Abomey-Calavi, Benin

\section{Tente Brice}

Laboratoire de Biogéographie et Expertise Environnementale (LABEE)

Département de Géographie et Aménagement du Territoire (DGAT),

Université d'Abomey-Calavi, UAC-Bénin

\begin{abstract}
The presence of a border promotes the development of trade between two states. Thus, cross-border trade is a highly developed activity in the five Communes of the Plateau Department, a Department bordering Nigeria on the entire eastern side. The present work aims to characterize the border area of the Plateau Department, in the south-east of Benin. To achieve the objectives relating to the description of economic activities and the main products traded in the study area, investigations were made from the collection of data and information by means of documentary research, direct observations in the field, interviews and surveys with 390 households of cross-border trade players as well as and interviews with 51 resource persons and authorities in the study environment. The results obtained were analyzed and interpreted using the SWOT model (Strengths, Weaknesses, Opportunities and Threats). The results obtained show that markets are the main places that structure the cross-border space of the Plateau Department. They are public, fixed and secure meeting places where buyers and sellers jostle each other and even compete fiercely on busy days. This activity is organized through the river transaction from Kétoukpè up to Banigbé in the Municipality of Ifangni and by land from Igolo up to Idigny in the Municipality of Kétou. The products traded are, in order of importance, food consumption products $(25.90 \%)$, nonfood manufactured products (22.89\%), petroleum products (19.74\%), chemicals (17.69\%), equipment products $(9.23 \%)$ and used vehicles $(4.62 \%)$.
\end{abstract}


Finally, this activity also has significant socio-economic effects.

Keywords: Plateau Department, border, trade, border area, products

\section{Introduction}

Les échanges commerciaux ont depuis des millénaires occupé une place de choix dans la vie de relation des populations. Cependant, ces échanges ont pris de l'ampleur à l'époque de la révolution industrielle durant laquelle la science et la technique ont donné les moyens de créer des richesses et de transporter les marchandises d'une partie du monde à une autre. Les peuples africains ne sont pas demeurés en marge de cette réalité (A. D. Aïchéou, 2017, p. 11).

L'histoire du commerce régional en Afrique de l'Ouest est celle d'une activité d'échanges impliquant les différentes zones écologiques. C'est aux frontières de ces zones écologiques que se sont développés depuis la période précoloniale les principaux marchés-entrepôts qui ont pendant longtemps garanti le dynamisme des échanges régionaux (J. Igué, 1993, p. 21). Le tracé actuel des frontières politiques de l'Afrique résulte d'un découpage de premier jet. Il a été calculé que $87 \%$ de la longueur de ces frontières, soit $70000 \mathrm{~km}$ sur un total d'environ $80000 \mathrm{~km}$, étaient directement hérités des partages coloniaux (M. Foucher, 1994, p. 167). Selon ABéGIEF (2013, p. 143), la frontière est un objet géographique séparant deux systèmes territoriaux contigus et ne se résume pas à une limite. Elle a des incidences sur l'organisation de l'espace (effets-frontière) et intègre une dimension politique (touche à la structuration d'une société).

Par ailleurs, la frontière a la capacité de faire émerger des pôles d'attraction qui exercent un effet magnétique sur les hommes et sur leurs activités. L'existence d'une frontière ne semble point être un obstacle encore moins un frein qui les empêche de se tourner les uns vers les autres. Cette ouverture a favorisé l'existence de relations plus ou moins intenses dans divers domaines. On les observe dans les domaines socioculturels et commerciaux ; mais les échanges commerciaux sont, sans conteste, les plus prépondérants. Ils concernent essentiellement les produits et marchandises, les denrées de première nécessité, des matériels divers: appareils électroniques et électroménagers (S. Diallo, 2006, p. 10).

Cependant, la mondialisation, les partenariats commerciaux, l'intégration économique sont autant d'éléments qui influencent les échanges entre les pays, et partant les performances économiques de chacun notamment captées à travers leur capacité à tirer le maximum de profit du commerce international. Dans cet élan, l'Union Africaine (UA), dans le cadre du renforcement de l'intégration économique entend porter le commerce intra africain de $13 \%$ à $25 \%$ des échanges du continent à l'horizon 2028. Ce taux 
ne prend pas en compte les échanges transfrontaliers non enregistrés, très dynamiques entre certains pays et qui pourraient considérablement modifier cette contribution (Institut National de la Statistique, 2014, p. 7).

L'analyse de la position géographique du Bénin, son armature urbaine et des dynamiques régionales ouest-africaines révèlent l'existence de cinq grands espaces frontaliers: l'espace bénino-nigérian, l'espace bénino-nigérien, l'espace bénino-burkinabè, l'espace bénino-togolais et la façade maritime (Schéma National d'Aménagement du Territoire, 2017, p. 78). Les frontières bénino-nigerianes en particulier ont été historiquement des territoires de concentration des activités économiques. Le sud-est est un exemple particulier grâce aux marchés locaux, mais aussi, ceux de Lagos et de Badagry qui furent des marchés coloniaux. La frontière entre le Bénin et le Nigeria est vitale pour les deux États, mais aussi pour la sous-région Ouest africaine. Elle facilite les échanges de toutes sortes par les diverses voies de communication : voie terrestre, voie fluviale et voie maritime. Cette vitalité explique la forte densité des activités économiques et le mouvement des personnes et des biens qui sont observés par les services des deux pays (L. Sossou-Agbo, 2011, p. 2).

La problématique des transactions entre le Bénin et le Nigeria concerne tous les Départements du Bénin dont celui du Plateau en particulier car sa situation géographique fait de lui une zone d'échanges commerciaux nationale et internationale. Le Département fait frontière dans sa partie est avec la République Fédérale du Nigeria. Il dispose de huit (8) marchés frontaliers. Ces marchés constituent un atout pour le département en ce sens que les échanges commerciaux qui s'y déroulent permettent aux communes de mobiliser des recettes pour financer le développement local. La dynamique commerciale entre les communes du Plateau et la République Fédérale du Nigeria laisse entrevoir une vie de relations qui met en jeu plusieurs acteurs (commerçants, autorités locales, agents contrôleurs, etc.) (S. Zannou, 2014, p. 76). La présente étude est entreprise dans le Département du Plateau. L'importance et la pertinence du choix dudit Département se justifient à double titre. Dans un premier temps, le contexte géographique du Département (frontière avec le Nigeria) et dans un second temps, le développement des activités de réexportation (de véhicule d'occasion, de céréales) et d'importation (produits manufacturés) ont motivé le choix de cette étude.

\section{Milieu d'etude}

Les marchés sont les principaux lieux qui structurent l'espace transfrontalier. Ce sont des lieux publics, fixes et sûrs de rencontre où acheteurs et vendeurs se bousculent voire rivalisent d'ardeur les jours d'animation. Les communes du département du Plateau comptent plusieurs marchés frontaliers illustrés sur la figure ci-dessous. 


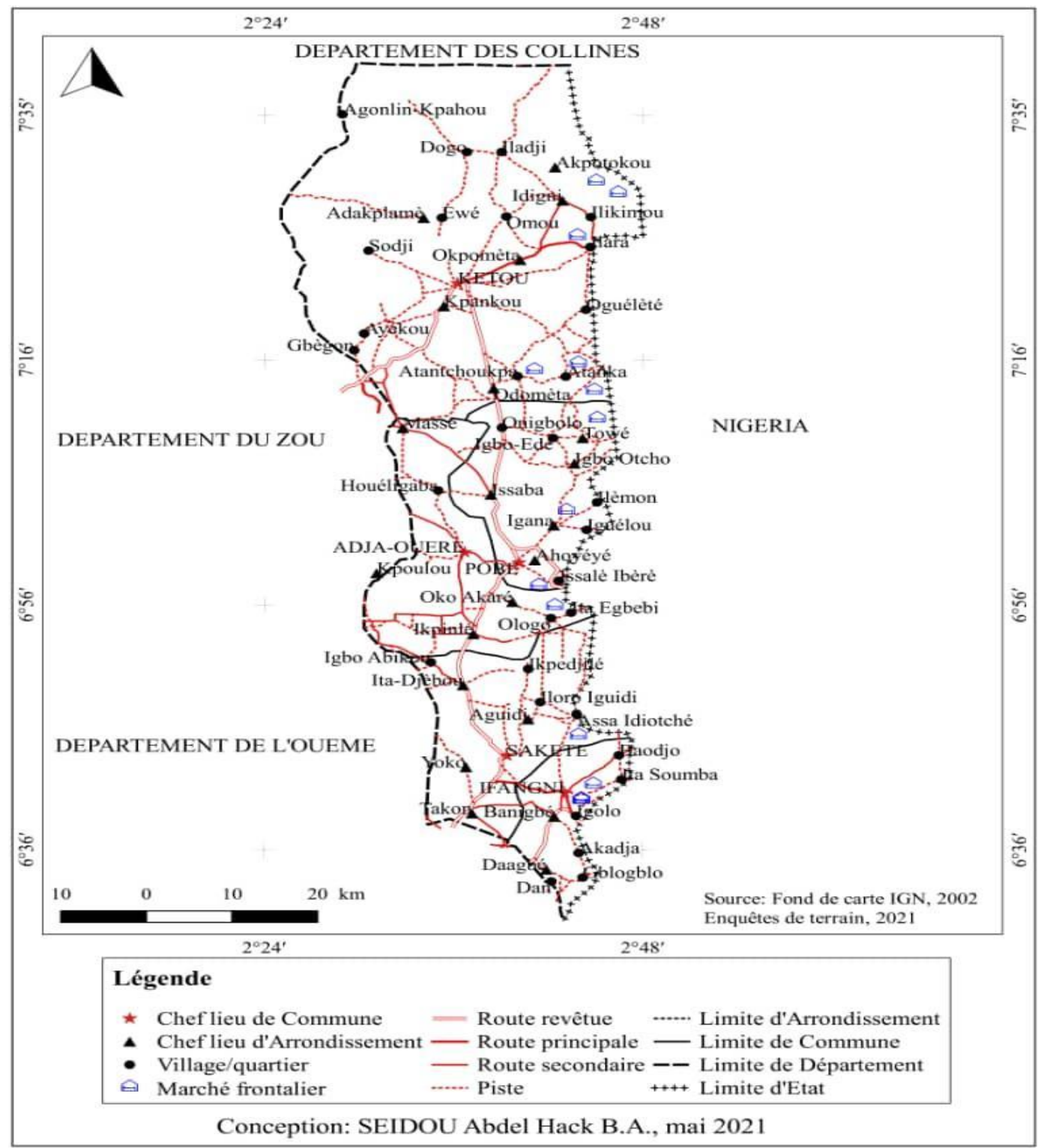

Figure 1 : Situation géographique des marchés frontaliers du département du Plateau

A la lecture de cette figure, il faut retenir que chacune des communes du département du Plateau dispose au moins d'un marché frontalier. Les marchés frontaliers existants se situent à une distance non éloignée de la frontière.

\section{Methodologie}


Plusieurs données ont été utilisées dans le cadre de cette recherche. Il s'agit entre autre des :

- données quantitatives relatives à la quantité des produits agricoles et marchandises ;

- données quantitatives relatives aux statistiques agricoles portant sur les superficies emblavées et les rendements des campagnes agricoles sur la période de 2010-2020, obtenues à la DDAEP / Plateau qui ont permis d'évaluer la part des produits agricoles dans les échanges transfrontaliers ;

- données quantitatives relatives à la quantité des principaux produits échangés entre le Bénin et le Nigeria dans le Département du Plateau qui ont permis de faire le sondage des divers produits échangés.

La carte ci-dessous présente la situation géographique des arrondissements frontaliers parcourus dans le cadre de cette thèse. 


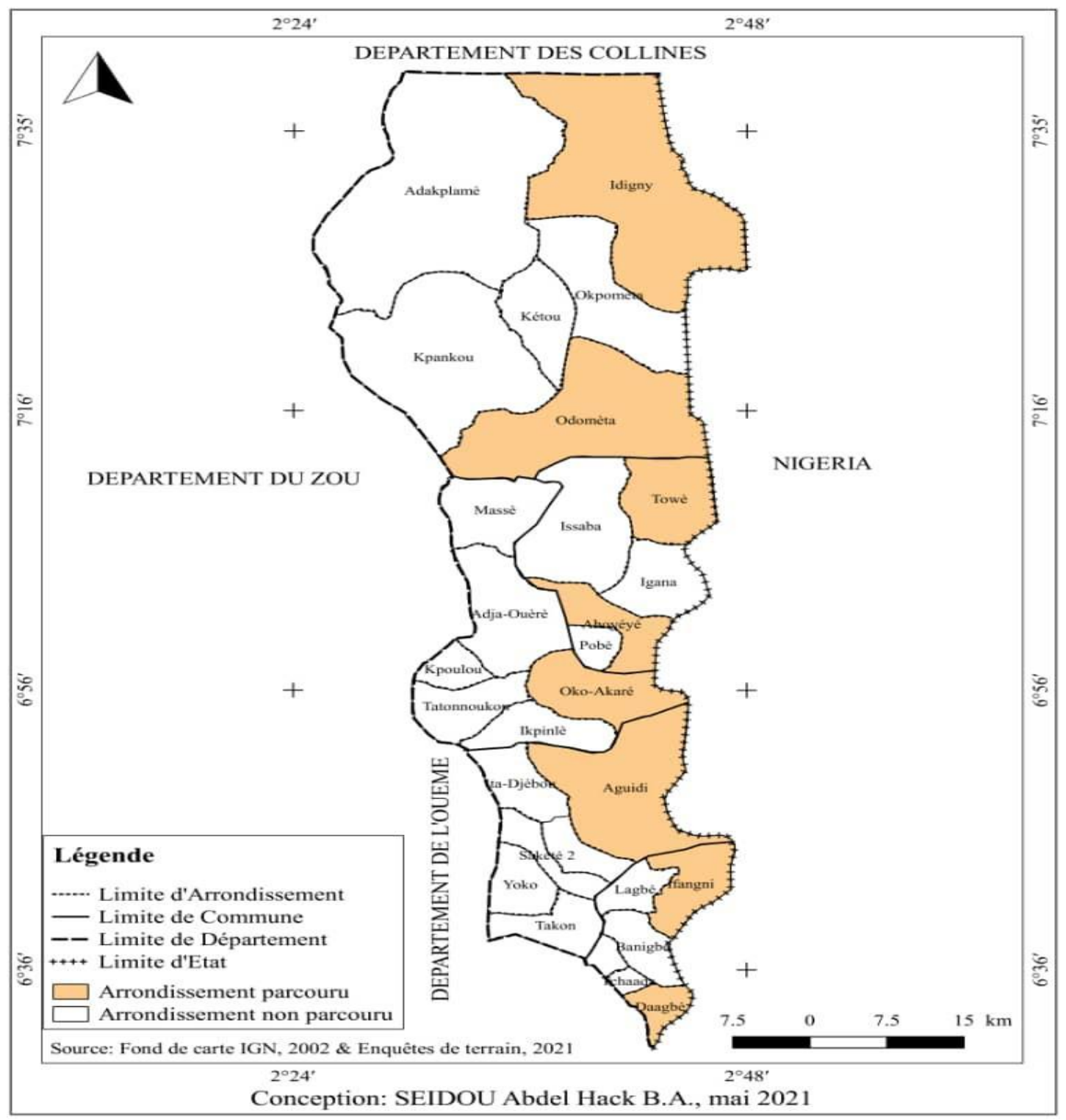

Figure 2 : Répartition des zones d'enquête dans les Communes d'étude

\section{Techniques de Collecte des Donnees}

La recherche documentaire a consisté à visiter les centres de documentation. Ainsi, la lecture de quelques ouvrages généraux et spécifiques, des mémoires, des revues et articles traitant des questions de frontière en général et du commerce transfrontalier en particulier a permis de faire le point de la documentation relative sur la problématique. 


\subsection{Enquetes de Terrain}

Pour mieux apprécier l'organisation des échanges dans cet espace géographique, les enquêtes ont été effectuées essentiellement au niveau des acteurs du commerce transfrontalier (commerçants, transporteurs, cambistes et passeurs) dans les communes du Département du Plateau. Ainsi donc, l'effectif des acteurs du commerce a été obtenu au niveau des associations de ces acteurs dans les arrondissements directement frontaliers avec le Nigeria. $\mathrm{Au}$ total huit arrondissement sont pris en compte pour l'enquête à savoir dans la Commune d'Ifangni : Daagbé, Banigbé et Ifangni ; dans la Commune de Sakété : Aguidi ; dans la Commune d'Ikpinlè : Oko-Akaré ; dans la Commune de Pobè : Towé et Ahoyéyé et enfin dans la Commune de Kétou : Odomèta et Idigny. Deux villages sont pris par arrondissements, sauf dans la Commune d'Ifangni où trois arrondissements ont été pris en compte, ce qui fait un total de huit (09) arrondissements et dix-sept (17) villages parcourus dans les cinq (05) Communes. La taille de l'échantillonnage pour cet objectif est déterminée par la méthode des quotas, qui est une méthode fondée sur le choix raisonné et le caractère représentatif est développé pour identifier les principaux acteurs. Le choix du groupe cible enquêté a répondu aux critères suivants :

- être un acteur exerçant une activité commerciale transfrontalière âgé de 18 au moins, ceci pour recueillir des informations raisonnables et fiables ;

- avoir exercé cette activité commerciale dans le secteur d'étude tout au moins les 05 dernières années afin de décrire la situation réelle de cette dernière ;

- être un transporteur des produits du commerce transfrontalier ayant au moins 5ans d'expérience dans le secteur, ceci pour donner des informations fiables à la présente étude ;

- être un passeur des produits du commerce transfrontalier ayant au moins 5 ans d'expérience dans le domaine, ceci pour également fournir des informations nécessaires et fiables à la présente étude.

Sur cette base, 230 acteurs (commerçants, transporteurs, passeurs et cambistes) sur les 362 enregistrés au niveau de leurs associations ont été enquêtés soit un total de $64 \%$ des acteurs.

\subsection{Traitement des Donnees et Analyse des Resultats}

Les résultats sont essentiellement présentés en tableaux de fréquences simples et en graphes. Pour la réalisation des graphiques, tableaux simples et croisés, les logiciels Word 2007 et Excel 2007 ont été utilisés. Ces différents tableaux et graphiques ont été commentés et analysés pour une meilleure visualisation des phénomènes et une bonne compréhension de l'idée du 
premier objectif. En effet, les graphes et tableaux ont l'avantage de permettre une lecture rapide des résultats.

Dans le but de caractériser la dynamique de l'espace transfrontalier du Département du Plateau, lieux des collectes de produits agricoles dans le secteur d'étude, une analyse des changements d'état a été nécessaire. Pour l'évaluation du couvert végétal des communes frontalières du département du Plateau, des cartes d'occupation et d'utilisation du sol sont nécessaires. Ces cartes ont été extraites d'image LANDSAT TM 1998 et d'image SENTINELLE 2018. L'évaluation des changements des unités d'occupation du sol a été faite à partir du taux d'expansion spatiale, du taux de conversion et de la matrice de transition.

\section{- Taux moyen annuel d'expansion spatiale}

Le taux moyen annuel d'expansion spatiale exprime la proportion de chaque unité de végétation naturelle qui change annuellement. Ce taux annuel (P. D. Kombienou, 2012, p. 78) Ta est calculé à partir de la formule suivante

$$
T a=\mathrm{S} 2-\mathrm{S} 1 \mathrm{~S} 1 \mathrm{x}(\mathrm{t2}-\mathrm{tl}) \times 100, \text { avec }
$$

S1 la superficie d'une unité de végétation à la date $\mathbf{t 1}, \mathbf{S 2}$ la superficie de la même unité de végétation à la date $\mathbf{t} \mathbf{2}$ et $\mathbf{t}$ le nombre d'années entre $\mathbf{t} \mathbf{1}$ et $\mathbf{t} \mathbf{2}$.

\section{- Taux de conversion}

Le taux de conversion d'une classe de végétation correspond au degré de transformation subie par cette classe de végétation en se convertissant vers d'autres classes. C'est alors la quantité de changements observés au niveau d'une formation végétale entre deux dates $\mathbf{t} \mathbf{0}$ et $\mathbf{t} \mathbf{1}$. Il permet ainsi de mesurer le degré de conversion d'une formation végétale en d'autres unités d'occupation du sol. Il s'obtient (P. D. Kombienou, 2012, p. 78) à partir de la matrice de transition suivant la formule :

$$
\mathrm{TC}=\Sigma \mathrm{ST}-\mathrm{Ss} \Sigma \mathrm{ST} \times 100 \text {, avec }
$$

TC le taux de conversion, ST les superficies des unités d'occupation du sol issues de la conversion d'une formation végétale, Ss la superficie de la même formation végétale demeurée stable à la date $\mathbf{t} 1$.

\section{- Matrice de transition}

Elle permet de mettre en évidence les différentes formes de conversion qu'ont subies les formations végétales entre deux instantanées. Elle est constituée de $\mathbf{X}$ lignes et de $\mathbf{Y}$ colonnes. Le nombre de lignes de la matrice indique le nombre de formations végétales au temps to ; le nombre $\mathbf{Y}$ de colonnes de la matrice est le nombre de classes de végétation converties au temps $\mathbf{t} 1$ et la diagonale contient les superficies des formations végétales 
restées inchangées. Les transformations se font donc des lignes vers les colonnes. Les superficies de ces différentes classes de végétation ont été calculées à partir du croisement des cartes de végétation de deux dates à l'aide de la fonction Intersect de la boîte à outils Arctoolbox du logiciel ArcGIS 10.4.

La démarche d'analyse des résultats est inscrite dans la logique du modèle SWOT : Strengths, Weaknesses, Opportunities and Threats (Forces, Faiblesses, Opportunités et Menaces).

\section{Resultats}

\subsection{Fonctionnement des marchés frontaliers dans les communes du département du Plateau}

Les principaux produits objet d'échanges transfrontaliers et le circuit des produits commercialisés permettent d'appréhender le fonctionnement des marchés dans les communes du département du Plateau.

Tableau 3 : Marchés frontaliers situés dans les communes du département du Plateau

\begin{tabular}{|c|c|c|c|c|}
\hline Communes & Arrondissement & Villages & Marché & $\begin{array}{l}\text { Estimation } \\
\text { de la distance } \\
\text { par rapport à } \\
\text { la frontière }\end{array}$ \\
\hline \multirow[t]{6}{*}{ Kétou } & \multirow[t]{3}{*}{ Odometa } & Atanchoukpa & Atanchoukpa & $7 \mathrm{~km}$ \\
\hline & & Atanka & Atanka & $5 \mathrm{~km}$ \\
\hline & & Tobolo & Tobolo & A $500 \mathrm{~m}$ \\
\hline & \multirow[t]{3}{*}{ Idigny } & Illara & IllaraKanga & A $300 \mathrm{~m}$ \\
\hline & & Iwoyé & Iwoyé & A $100 \mathrm{~m}$ \\
\hline & & Iwoyé & Marché à bétail & A $500 \mathrm{~m}$ \\
\hline \multirow[t]{3}{*}{ Pobè } & Iganan & Iganan & Iganan & $4 \mathrm{~km}$ \\
\hline & Ahoyéyé & Issalè-Ibéré & Abè-Ekpè & A $600 \mathrm{~m}$ \\
\hline & Towé & Igbôkôfi & Igbôkôfi & A $300 \mathrm{~m}$ \\
\hline Sakété & Aguidi & Assa-Idioché & Modogan & A $400 \mathrm{~m}$ \\
\hline Adja-Ouèrè & Oko-Akaré & $\begin{array}{l}\text { Etaègbèbi- } \\
\text { Adéromou }\end{array}$ & Ilouda & A $550 \mathrm{~m}$ \\
\hline \multirow[t]{3}{*}{ Ifangni } & \multirow[t]{3}{*}{ Ifangni } & $\begin{array}{c}\text { BB (Bénin } \\
\text { Border) }\end{array}$ & BB & A $100 \mathrm{~m}$ \\
\hline & & Igolo & Igolo & $\mathrm{A} 100 \mathrm{~m}$ \\
\hline & & Ifangni & Ifangni & $2 \mathrm{~km}$ \\
\hline
\end{tabular}

Source : Travaux de terrain, 2021

De l'analyse du tableau 3, il faut noter que chaque commune dispose d'au moins un marché frontalier. La commune de Kétou représente la première commune qui regorge d'un nombre important de marché frontalier qui s'élève à six (06) marchés. Les communes de Pobè et d'Ifangni viennent en deuxième position et compte trois (03) marchés frontaliers chacune. Alors que les communes de Sakété et d'Adja-Ouèrè comptent chacune un marché frontalier. Il est à noter que chaque marché frontalier s'anime en moyenne dans un intervalle de quatre jours soit huit fois par mois. Ces marchés sont situés non 
loin des principales voies d'accès et installés sur de grande superficie avec des boutiques ou hangars construits en matériaux précaires ou définitifs. La fréquentation de ces marchés et le nombre important de produits qui y transitent permettent aux populations de satisfaire leurs diverses demandes. En sus, les zones frontalières abritant ces marchés constituent des points d'ancrage ou de relais des transactions internationales.

Les photos de la planche ci-dessous montrent l'aspect du grand marché de bétails à Iwoyé dans l'arrondissement d'Idigny, Commune de Kétou.

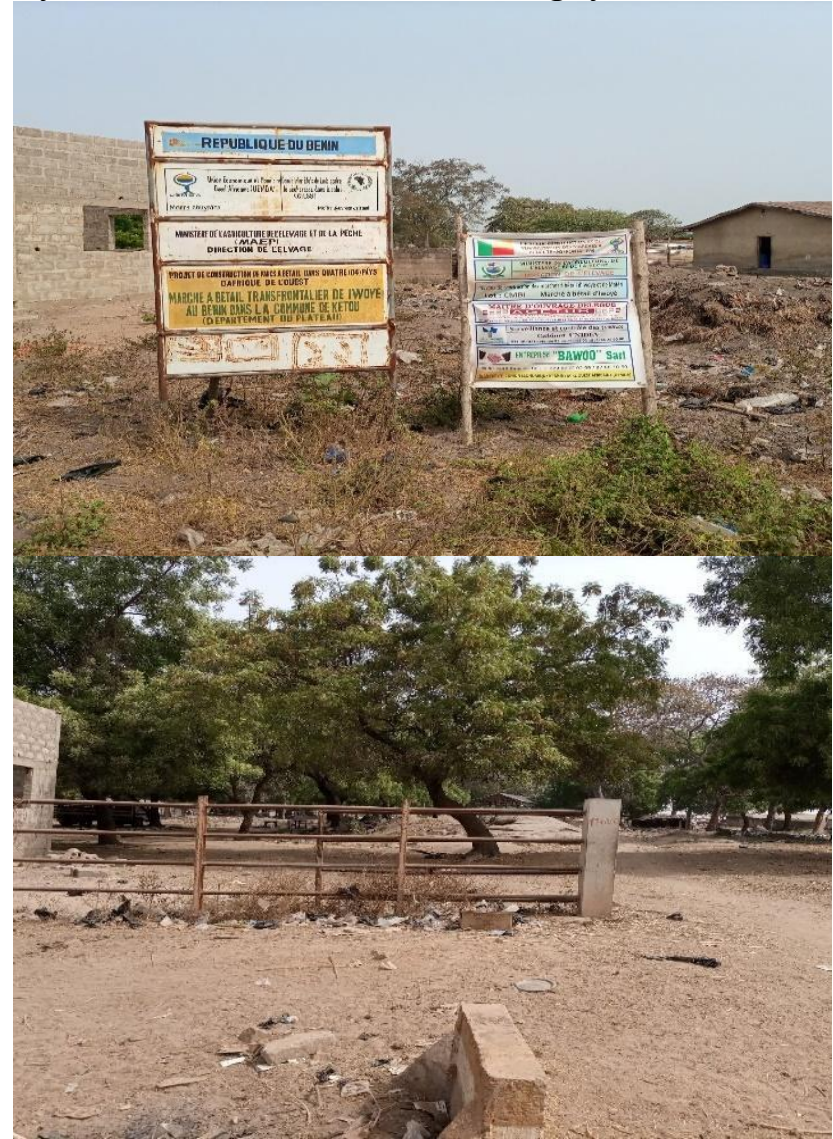

Planche 1 : Marché à bétails transfrontalier à Iwoyé dans l'arrondissement d'Idigny Prise de vue : Seidou, décembre 2020

Les deux photos de la planche 1 présentent l'aspect du grand marché à bétails transfrontalier à Iwoyé l'arrondissement d'Idigny. C'est l'un des plus grands marchés à bétails du Bénin et de la sous-région, construit sous financement du projet de construction et de réhabilitation des marchés à bétails transfrontalier de l'UEMOA. Ce marché situé à la frontière même est un point stratégique d'échange et de brassages culturels. Il devient de plus en plus important à travers l'effectif des commerçants et des acheteurs qu'il reçoit (50 
à 90 vendeurs pendant les jours de grandes affluences). D'autre part, ces divers marchés constituent une source de mobilisation des ressources pour les communes qui les abritent.

\section{- Entrepôts}

Dans les communes frontalières, il importe de préciser l'existence des magasins ou entrepôts de stockage de gros volumes de marchandises, destinées à franchir la frontière. Ces entrepôts frontaliers sont consacrés à la revente pour les grands commerçants. La technique d'observation directe a permis de répertorier le nombre d'entrepôts au niveau des espaces frontaliers du Département du Plateau. Le tableau 4 ci-dessous permet de faire le point de ces entrepôts.

Tableau 4 : Point des entrepôts au niveau de l'espace frontalier du Département du Plateau

\begin{tabular}{|c|c|c|c|}
\hline Communes & Arrondissements & Espace frontalier & $\begin{array}{c}\text { Nombre } \\
\text { d'entrepôts }\end{array}$ \\
\hline \multirow{3}{*}{ Ifangni } & Daagbé & Gbloblo, Daagbé & 06 \\
\cline { 2 - 4 } & Ifangni & Igolo, Baodjo & 22 \\
\cline { 2 - 4 } & Banigbé & Banigbé-Nagot & 10 \\
\hline Sakété & Aguidi & Modogan & 12 \\
\hline Adja-Ouèrè & Oko-Akaré & Ologo, Iwinka & 15 \\
\hline Pobè & Towé & Towé & 08 \\
\cline { 2 - 4 } & Ahoyéyé & Okéita & 18 \\
\hline Kétou & Idigny & Illara, IIwoyé & 25 \\
\hline
\end{tabular}

Source : Travaux de terrain, 2021

L'observation effectuée le long du périmètre des espaces frontaliers de chaque commune du Département du Plateau révèle après analyse du tableau 4 que le secteur Illara- Iwoyé comporte le plus grand nombre d'entrepôts avec 25 recensés. On a ensuite le secteur Igolo- Baodjo avec 22 entrepôts; le secteur de Okéita et de Towé dans la commune de Pobè avec 26 entrepôts ; le secteur de Ologo- Iwinka avec 15 entrepôts recensés ; le secteur de Modogan dans la commune de Sakété avec 12 entrepôts. Cela confirme la densité des flux au niveau de ces espaces frontaliers. Le nombre d'entrepôts au niveau de cet espace frontalier est en pleine croissance car beaucoup sont en cours de construction étant donné que cela permet à leur propriétaire de faire de bons bénéfices

La planche 2 montre les magasins de stockage des produits destinés aux échanges transfrontaliers. 


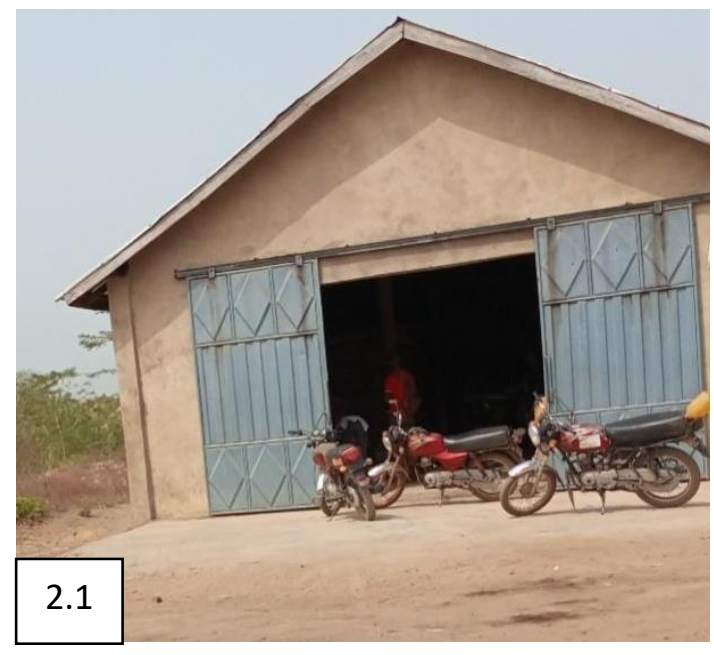

Grand magasin d'entreposage de riz à Iwoyé

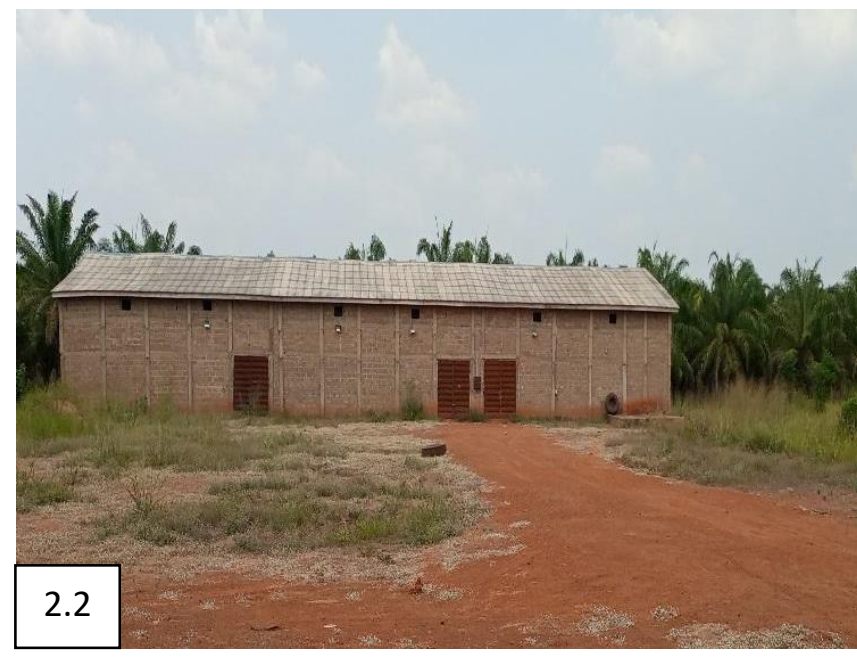

Magasin en construction à Oké-Ita non loin de la frontière

Planche 2 : Magasin de stockage des produits destinés aux échanges transfrontaliers

Prise de vue : Seidou, décembre 2020

La photo 2.1 est une illustration d'un grand magasin d'entreposage de riz à Iwoyé dans la Commune de Kétou et la photo 2.2, celle d'un magasin en construction à Oké-Ita dans la Commune de Pobè. Ces magasins servent de lieu de stockage de riz et d'huiles végétales provenant du port de Cotonou. On observe à la devanture du magasin des motos. Ce sont celles des personnes qui font le déchargement du riz et ces motos peuvent aussi servir dans le transport des marchandises en cas de fermeture de la frontière poreuse. Il existe plusieurs magasins de stockage de ces produits dans le secteur d'étude.

\subsection{Principaux produits objets d'échanges transfrontaliers}

Situées à des dizaines de kilomètres de la capitale économique et celle politique du Bénin, les communes du département du Plateau obtiennent une quantité importante de ses produits à travers les échanges frontaliers avec le Nigeria. Il convient de préciser qu'une kyrielle de produits font l'objet d'échanges transfrontaliers et ont pour noms : les produits de consommation alimentaire, les produits manufacturés non alimentaires, les produits d'équipement et des biens matériels, les véhicules d'occasion, les produits pétroliers et les produits chimiques. La figure 3 présente l'essentiel des produits qui font l'objet d'échanges transfrontaliers. 


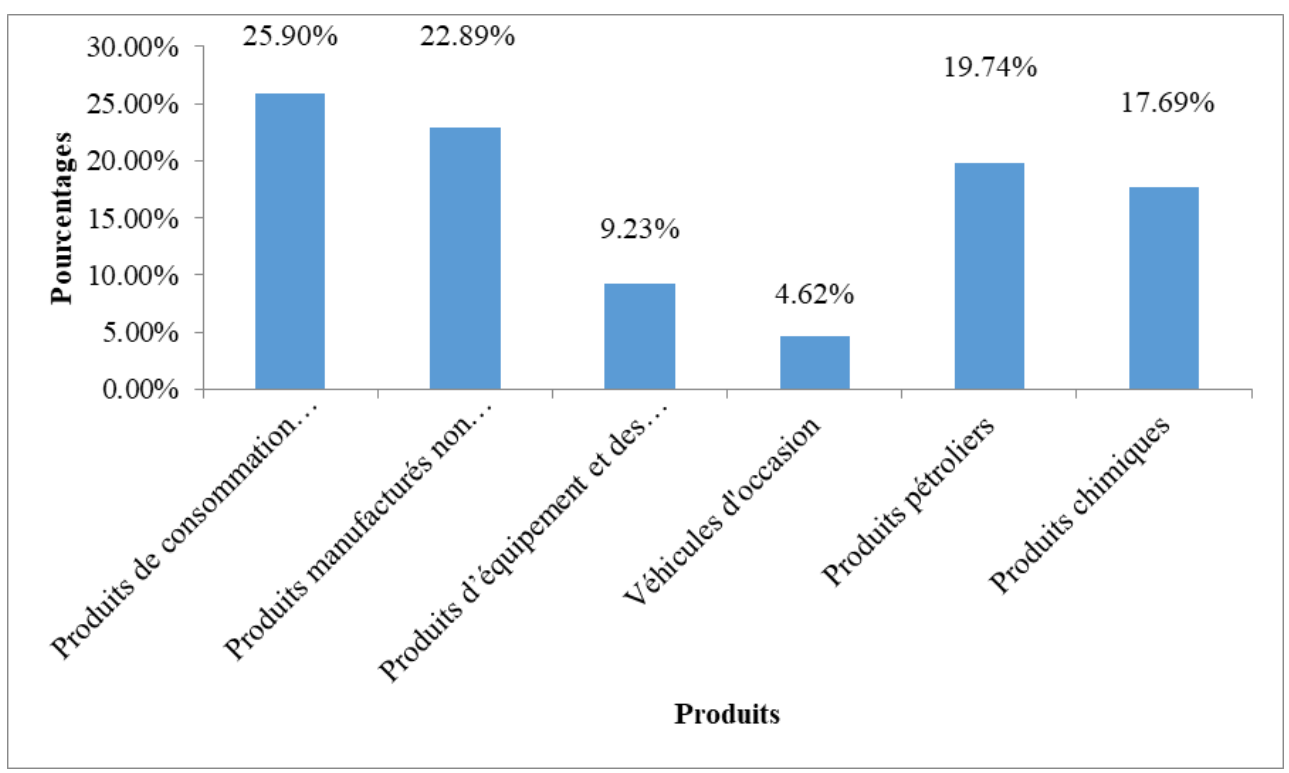

Figure 3 : Principaux produits objets d'échanges transfrontaliers

Source : Travaux de terrain, 2021

A la lecture de la figure 3 , il faut retenir que les produits échangés sont par ordre d'importance les produits de consommation alimentaire $(25,90 \%)$, les produits manufacturés non alimentaires $(22,89 \%)$, les produits pétroliers $(19,74 \%)$, les produits chimiques $(17,69 \%)$, les produits d'équipement $(9,23$ $\%)$ et les véhicules d'occasion $(4,62 \%)$.

Les enquêtes réalisées auprès des agents de la douane au niveau de la Direction Départementale a permis de recenser la liste des principaux produits objet d'échanges entre le Bénin et le Nigeria au niveau des postes de douane du Département du Plateau. Le tableau 5 présente la liste de ces produits dédouanés.

Tableau 5 : Principaux produits dédouanés au niveau des postes de douane du Département du Plateau

\begin{tabular}{|c|c|}
\hline Produits exportés vers le Nigeria & Produits importés du Nigeria \\
\hline Volaille & Boissons nigérianes \\
Riz & Ouvrages en matière plastique \\
Véhicules & Huile à moteur \\
Huiles végétales & Carreaux \\
Poissons congelés & Biscuits \\
Farine de blé & Motocycles \\
Pâtes alimentaires & Essence \\
Friperies & Gasoil \\
Sucre & Gaz \\
& Pétrole lampant \\
\hline
\end{tabular}

Source : Données Direction Départementale des douanes O/P, 2017 
L'analyse de ce tableau montre que la majorité des produits dédouanés et qui sont exportés vers le Nigeria sont ceux qui à leurs tours sont exportés vers le Bénin par les pays européens et asiatiques donc des produits qui ne sont pas fabriqués au Bénin. Dans le cadre de l'exécution des décisions contenues dans le mémorandum de Badagry signé le 14 août 2003 et en application de l'accord quadripartite conclu entre le Bénin, le Ghana, le Nigeria et le Togo, ces divers produits font d'ailleurs partir de la liste de produit prohibé à l'importation au Nigeria. Par contre tous les produits importés du Nigeria au Bénin à savoir : les boissons, les ouvrages en matières plastiques, les carreaux, l'essence, le gasoil, etc. sont des produits typiques du Nigeria. Cela certifie le statut de grand pays industrialisé et de première puissance économique en Afrique que représente le Nigeria.

\subsubsection{Produits de consommation alimentaire}

Les produits de consommation alimentaire portent essentiellement sur les produits agricoles et ses dérivés, les produits halieutiques, d'élevages et denrées alimentaires. La figure 4 décrit les catégories de produits de consommation alimentaire et leur proportion.
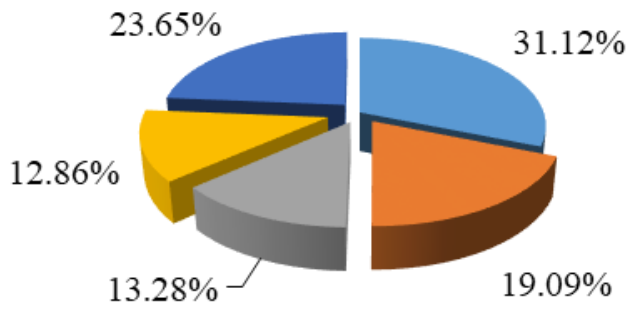

- Produits agricoles

- Dérivés des produits agricoles

- Produits halieutiques

Produits d'élevage

- Denrées alimentaires

Figure 4 : Proportion des produits de consommation alimentaire

Source : Travaux de terrain, 2021

L'analyse de la figure 4 illustre respectivement les produits alimentaires par ordre d'importance d'échange que sont les produits agricoles $(31,12 \%)$, les denrées alimentaires $(23,65 \%)$, les dérivés de produits agricoles $(19,09 \%)$, les produits halieutiques $(13,28 \%)$ et les produits d'élevage (12, $86 \%)$. 


\subsubsection{Produits agricoles}

L'économie du secteur d'étude est basée essentiellement sur les activités agricoles. La production est composée pour l'essentiel des cultures vivrières telles que manihot esculenta, zea mays, vigna unguiculata, capsicum annuum, solanum lycopersicum, les cultures de rente telles que elaeis guineensis. Ces divers produits sont exportés vers le Nigeria par les commerçants du département et en retour d'autres produits agricoles tels que solanum tuberosum et allium cepa sont également importés au Bénin. Le tableau 6 présente l'évolution des principaux produits agricoles dans les cinq Communes du Département du Plateau.

\begin{tabular}{|c|c|c|c|c|c|c|c|c|c|c|}
\hline & \multicolumn{10}{|c|}{ Production en tonnes } \\
\hline $\begin{array}{c}\text { Année } \\
\text { Produits }\end{array}$ & $\begin{array}{l}2010- \\
2011\end{array}$ & $\begin{array}{l}2011- \\
2012\end{array}$ & $\begin{array}{l}2012- \\
2013\end{array}$ & $\begin{array}{l}2013- \\
2014\end{array}$ & $\begin{array}{l}2014- \\
2015\end{array}$ & $\begin{array}{l}2015- \\
2016\end{array}$ & $\begin{array}{l}2016- \\
2017\end{array}$ & $\begin{array}{l}2017- \\
2018\end{array}$ & $\begin{array}{c}2018- \\
2019\end{array}$ & $\begin{array}{c}2019- \\
2020\end{array}$ \\
\hline Maïs & 80017 & 134306 & 152642 & 173460 & 189779 & 173690 & 186808 & 226639 & 213346 & $\begin{array}{l}218 \\
428\end{array}$ \\
\hline Manioc & 743618 & 848513 & 553111 & 832107 & 739257 & 780546 & 913437 & $\begin{array}{c}1224 \\
493\end{array}$ & $\begin{array}{c}1013 \\
705\end{array}$ & $\begin{array}{c}1068 \\
629\end{array}$ \\
\hline Igname & 22760 & 129493 & 24327 & 27179 & 30833 & 30098 & 28054 & 59601 & 39433 & 55648 \\
\hline Patate douce & 2609 & 3604 & 4160 & 367 & 341 & 733 & 1562 & 647 & 722 & 1747 \\
\hline Tomate & 22785 & 2647 & 30043 & 35843 & 35092 & 33824 & 36226 & 34759 & 36998 & 36626 \\
\hline
\end{tabular}

Tableau 6 : Evolution de la production agricole dans les communes de Plateau de 2010 à 2020

Source : DDAEP/ Plateau, 2021 
La demande nigériane a un effet dopant sur les exportations agricoles du Bénin et notamment sur l'offre frontalière. Pour satisfaire cette demande en pleine croissance liée à l'accroissement de la population, au développement des fermes d'élevage et à l'expansion du secteur agro-industriel, les commerçants nigérians mettent en place une stratégie proactive sur la production en faisant des achats anticipés sur la base de contrat de livraison. Disposant des capitaux ou des fonds de roulement plus importants que les commerçants béninois, ils contractent directement avec les producteurs en début de campagne pour une fourniture plus sure de leurs approvisionnements. Les prix contractuels proposés sont très incitatifs et plus élevés que les prix futurs du marché. Ainsi plusieurs espaces sont sollicités au niveau des espaces frontaliers pour la production agricole.

\subsubsection{Effets de la production agricole sur l'occupation de l'espace frontalier de 1998 à 2018}

Les changements spatio-temporels des unités d'occupation des terres dans le Département du Plateau ont été évalués à travers les cartes d'occupations des terres de 1998 (figure 5) à 2018 (figure 6).

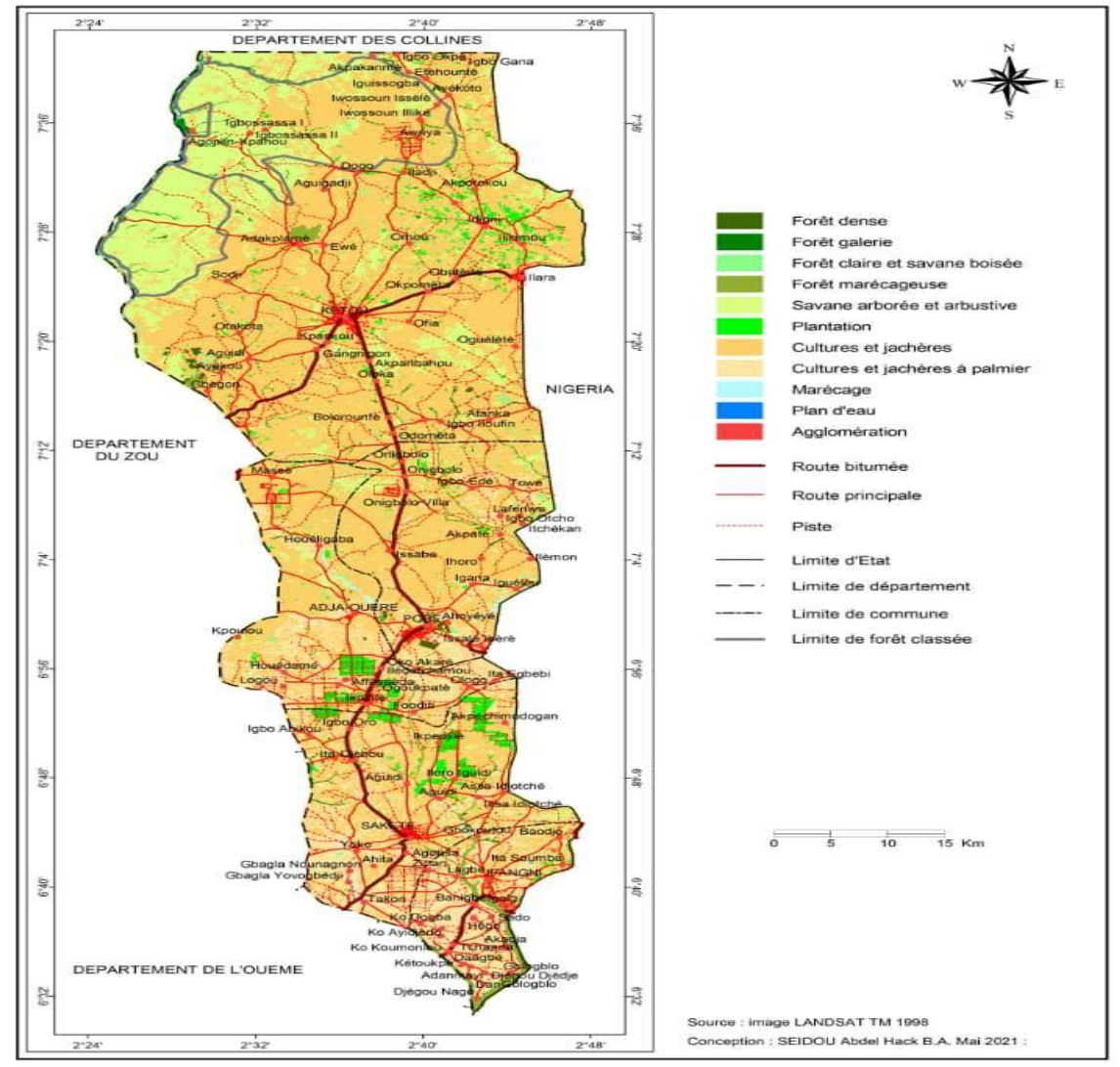

Figure 5 : Occupation des terres du Département du Plateau en 1998 


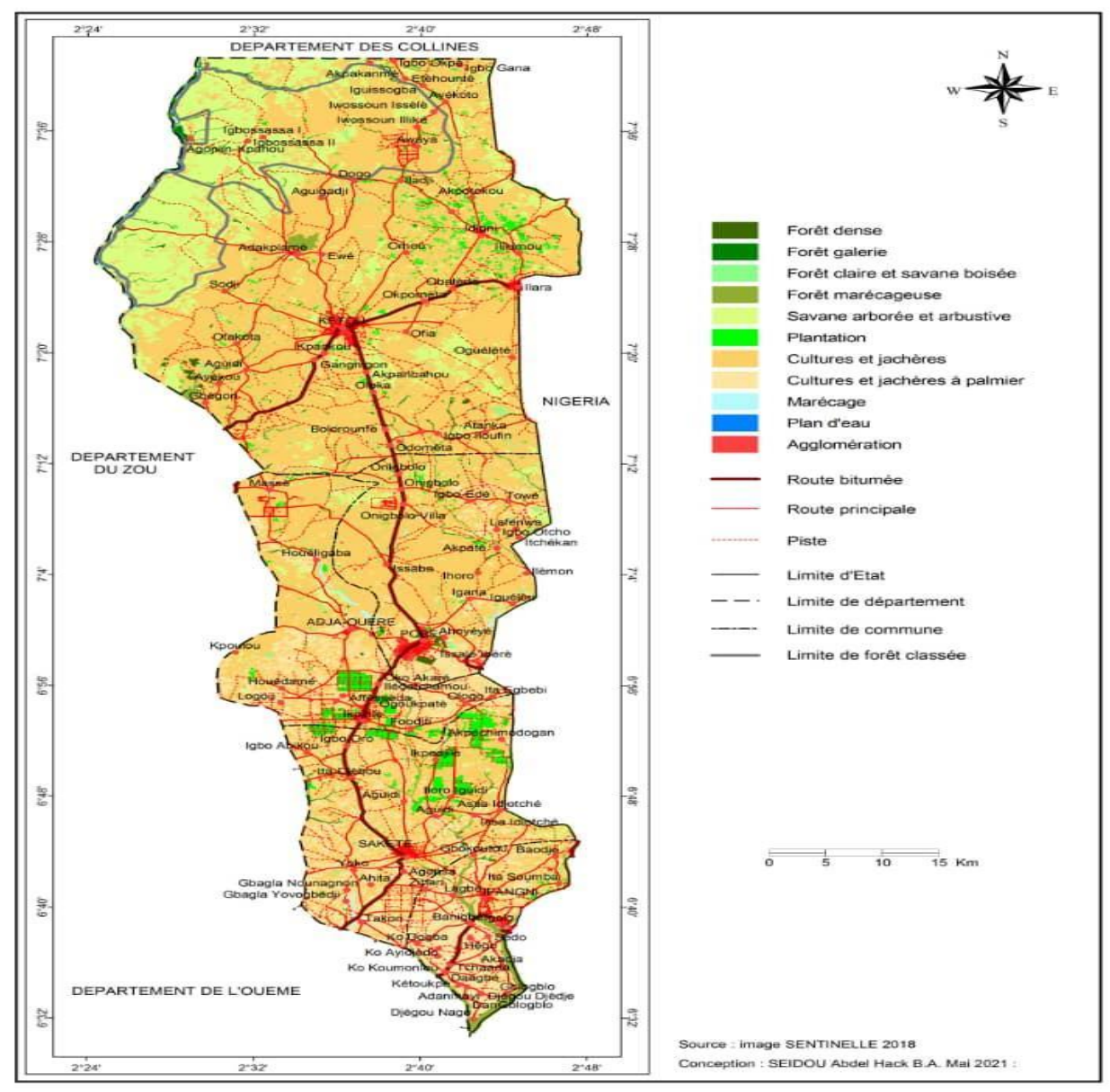

Figure 6 : Occupation des terres du Département du Plateau en 2018

L'examen des cartes d'occupation des terres entre 1998 et 2018 dans le Département du Plateau a permis de distinguer 11 classes d'occupation des terres (figure 4 et figure 5). Sur la carte d'occupation des terres en 1998 (figure 4), les unités sont composées des galeries forestières observées $(3111 \mathrm{Ha})$, des forêts claires et savanes boisées retrouvées $(1038 \mathrm{Ha})$, des forêts marécageuses $(3523 \mathrm{Ha})$, des savanes arborées et arbustives (49563 Ha). Les plantations (1066 Ha), la forêt dense (1175 Ha), les plans d'eau (516 Ha), les marécages (767 Ha), ainsi que les mosaïques de cultures et jachères $(219064 \mathrm{Ha})$, les mosaïques de cultures et jachères à palmier $(45291 \mathrm{Ha})$ et les agglomérations (1286 Ha). Contrairement à l'analyse de la carte d'occupation des terres de 2018 (figure 5), les unités sont composées des galeries forestières observées (3022 Ha), des forêts claires et savanes boisées retrouvées $(1092 \mathrm{Ha})$, des forêts marécageuse $(3504 \mathrm{Ha})$, des savanes arborées et arbustives $(48472 \mathrm{Ha})$. Les plantations $(1529 \mathrm{Ha})$, la forêt dense $(1092 \mathrm{Ha})$, les plans d'eau $(516 \mathrm{Ha})$, 
les marécages $(753 \mathrm{Ha})$, ainsi que les mosaïques de cultures et jachères (220414 Ha), les mosaïques de cultures et jachères à palmier (43702 Ha) et les agglomérations (3102 Ha). L'occupation des terres entre 1998 et 2018 a montré une forte anthropisation des écosystèmes naturels. De même au niveau des mosaïques de cultures et jachères et des plantations, on note une nette progression des surfaces cultivables en 2018 par rapport à 1998. Cela atteste de la forte productivité agricole du secteur d'étude. Le taux moyen annuel d'expansion spatiale et le taux de conversion des unités d'occupation des terres ont été synthétisés par le tableau 7.

Tableau 7 : Taux moyen annuel d'expansion spatiale et taux de conversion des unités d'occupation des terres de 1998 à 2018

\begin{tabular}{|c|c|c|c|c|}
\hline OCCUPATION DU SOL & $\mathbf{1 9 9 8} \mathbf{~ H a}$ & $\mathbf{\%}$ & $\mathbf{2 0 1 8} \mathbf{H a}$ & $\mathbf{\%}$ \\
\hline Forêt galerie & 3111 & $0,95 \%$ & 3022 & $0,93 \%$ \\
\hline Forêt dense & 1175 & $0,36 \%$ & 1092 & $0,33 \%$ \\
\hline Forêt claire et savane boisée & 1038 & $0,32 \%$ & 294 & $0,09 \%$ \\
\hline Forêt marécageuse & 3523 & $1,08 \%$ & 3504 & $1,07 \%$ \\
\hline Savane arborée et arbustive & 49563 & $15,18 \%$ & 48472 & $14,85 \%$ \\
\hline Plantation & 1066 & $0,33 \%$ & 1529 & $0,47 \%$ \\
\hline Cultures et jachères & 219064 & $67,12 \%$ & 220414 & $67,53 \%$ \\
\hline Cultures et jachères à palmier & 45291 & $13,88 \%$ & 43702 & $13,39 \%$ \\
\hline Marécage & 767 & $0,23 \%$ & 753 & $0,23 \%$ \\
\hline Plan d'eau & 516 & $0,16 \%$ & 516 & $0,16 \%$ \\
\hline Agglomération & 1286 & $0,39 \%$ & 3102 & $0,95 \%$ \\
\hline TOTAL & 326400 & $100,00 \%$ & 326400 & $100,00 \%$ \\
\hline
\end{tabular}

Source : Images TM 1998 et Images SENTINELLE 2018

L'analyse de ce tableau révèle une nette régression en 2018 des forêts galeries $(-0,02 \%)$, forêts denses $(-0,03 \%)$, forêt claire et savane boisée ($0,23 \%)$, des forêts marécageuses $(-0,01 \%)$, des savanes arborée et arbustive $(-0,33 \%)$ ainsi que des cultures et jachères à palmier (- $0,49 \%)$. A l'opposée, on note une progression au niveau des plantations $(+0,14 \%)$, des cultures et jachères $(+0,41 \%)$, des agglomérations $(+0,56 \%)$ et une stabilité au niveau des plans d'eau et marécage par rapport à l'année 1998. Toutes les unités d'occupation des terres ont connu une modification de leurs superficies selon qu'elles aient été une formation naturelle ou des formations anthropiques. La plus grande réduction a été observée au niveau des cultures et jachères à palmier à cause de cette guerre entre les Coopératives d'Aménagement Rural (CAR) qui a contribué à la destruction de plusieurs hectares de palmeraies du département du Plateau et la plus grande progression est au niveau des agglomérations.

En conclusion, ces analyses ont permis de dégager des changements majeurs dans le secteur d'étude à savoir: la dégradation de formations forestières, marquée par une réduction de superficies de forêt claire et savane 
boisée et de savane arborée et arbustive pour l'installation humaine et l'élargissement des surfaces cultivables au niveau du secteur d'étude.

\section{Discussion}

Plusieurs travaux de recherche ont porté sur la problématique du commerce transfrontalier en Afrique en général et au Bénin en particulier. Ils ont abouti pour la plupart à des résultats similaires que la présente recherche. C'est ainsi que les travaux de O. Walther et M. Kone (2018) à travers une analyse empirique des réseaux marchands organisés autour du textile d'occasion au sud du Niger ont montré que l'émergence du commerce de réexportation repose sur la proximité de la région au port de Cotonou et des conditions fiscales attrayantes pour mettre à profit la situation frontalière des marchés. Ils ajoutent dans leur résultat que les marchands du commerce de réexportation se sont installés au cours de trois grandes vagues de migration dont les origines géographiques sont toujours plus lointaines. Cependant, ils confirment que depuis l'élection du Président Buhari au Nigeria, l'essor de ce commerce de réexportation est toutefois remis en cause par les contrôles renforcés visant à limiter l'importation de produits frappés d'interdiction.

Cette étude, à l'instar de celle menée par Kombiéni et al (2021, p. 61) au Nord-Ouest de l'Atacora au Bénin révèlent que les échanges dans cet espace frontalier sont à $92 \%$ commerciaux et $8 \%$ à but touristique. Ils ajoutent que toutefois, $91 \%$ des produits agricoles de cet espace sont collectés dans les différents marchés des Communes frontalières de l'Atacora vers le BurkinaFaso contre $9 \%$ d'exportation de l'essence de contrebande et d'importation des produits manufacturiers. Pour finir, ils confirment que ces activités commerciales ont des impacts important car ils génèrent des revenus aux acteurs du secteur d'étude et contribuent ainsi au développement local des Communes frontalières de l'Atacora à travers le paiement des taxes et impôts.

De même, K. Titeca et C. Kimanuka (2012) trouvent que le commerce informel transfrontalier joue un rôle considérable dans la région des Grands Lacs, où des grandes quantités de biens sont échangées par les petits commerçants entre la République Démocratique du Congo, le Rwanda, l'Ouganda et le Burundi, permettant l'approvisionnement des biens particuliers (surtout des produits vivriers) pour des régions qui ne produisent pas assez pour couvrir leur besoin.

Par ailleurs, l'ABéGIEF (2015, p. 1) à travers le Programme National de Gestion Intégrée des Espaces Frontaliers du Bénin propose de renforcer la souveraineté nationale dans les zones frontalières afin de sécuriser le territoire national et de créer de meilleures conditions d'existence aux populations desdites zones. Spécifiquement, ce programme veut faire des espaces frontaliers de véritables zones de développement local concourant à la lutte contre la pauvreté et à l'amélioration des conditions de vie des populations. 
Ces différents résultats s'apparentent à ceux de cette recherche. En effet, l'espace frontalier du département du Plateau au Sud-est du Bénin dispose de plusieurs marchés frontaliers qui permettent l'écoulement des divers produits d'échanges entre le Bénin et le Nigeria.

\section{Conclusion}

Au terme de cette étude, les principaux atouts naturels et humains ont permis d`appréhender l'importance des échanges commerciaux frontaliers dans le Département du Plateau au Sud-est du Bénin. En étudiant ces différentes caractéristiques dont la plupart des activités transfrontalières échappent au contrôle des Etats et sont le fait des individus et de réseaux plus ou moins organisés. Ces activités révèlent le fait que, les populations frontalières en général et celle du département du Plateau et au Nigéria en particulier, ont le souci de leur épanouissement quotidien et n'attendent pas nécessairement les ressources mises à leur profit par les gouvernants mais essayent de profiter des richesses créées par leurs Etats ou venant d'ailleurs.

\section{References:}

1. Agence Béninoise de Gestion Intégrée des Espaces Frontaliers (ABéGIEF), 2013, Politique Nationale de développement des espaces frontaliers. MISPC, Bénin, $231 \mathrm{p}$.

2. Agence Béninoise de Gestion Intégrée des Espaces Frontaliers (ABéGIEF), 2015, Association Béninoise des Communes Frontalières $(\mathrm{ABCF})$ : un outil stratégique dans la gestion des frontières, Agenda des frontières 2015. http://www.abegief.org/?webmater-ABeGIEF, (consulté le12/01/2021à16h).

3. AÏCHEOU Dossa Alfred, 2017, Marché des produits agricoles vivriers du Département de l'Atlantique au Sud du Bénin : fonctionnement, implication économiques et spatiales. Mémoire de Thèse de Géographie, EDP/FLASH/UAC, 315 p.

4. DIALLO Souleymane, 2006, Dynamiques transfrontalières et développement local urbain dans un contexte de décentralisation : le cas de la commune de Rosso Sénégal. Saint-Louis, Université Gaston Berger. Mémoire de DEA, $112 \mathrm{p}$.

5. DOMINGO Etienne, 2008, La région urbaine du littoral du Bénin : dynamiques urbanisantes et environnement, une géographie de l'aménagement du territoire. Thèse de doctorat d'Etat de Géographie. Université de Lomé, 585 p.

6. FOUCHER Michel, 1994, Fronts et frontières. Fayard, Paris, 692 p.

7. IGUE Ogunsola John, 1993, Echanges et espaces de développement : cas de l'Afrique de l'Ouest. Travaux de l'Institut de Géographie de Reims, n 83-84, 1993, 19-39. 
8. Institut National de la Statistique (INS), 2014, Enquête sur les échanges transfrontaliers de marchandises au Cameroun : Bilan méthodologique et résultats. Rapport de transaction, 2014. 60 p.

9. KOMBIENI M'Bouaré Frédéric, KOMBIENI Azouma Hervé, ASSOUNI Janvier et TADJA Idrissou, 2021, Echanges transfrontaliers entre les communes frontalières au Nord-Ouest de l'Atacora au Bénin et le Burkina-Faso. Journal of Research in Humanities and Social Science, Vol 9, ISSN : 2321-9467, 61-74.

10. KOMBIENOU Pocoun Damè, 2012: Influences des systèmes de productions agricoles sur l'occupation des terres, la fertilité des sols et l'agro-biodiversité en zone montagneuse au nord-ouest du Bénin. Thèse de doctorat, EDP/FLASH/UAC, 291 p.

11. Schéma National d'Aménagement du Territoire (SNAT). Edition septembre 2017, 175.

12. SOSSOU-AGBO Lazare, 2011, Dynamique territoriale à la frontière bénino-nigeriane : Rôle des marchés du Sud-Est. Brit XI, Sep 2011. Genève/ Grenoble, France, 23 p.

13. TITECA Kristof et KIMANUKA Célestin, 2012, Marché dans l'obscurité : le commerce informel transfrontalier dans la région des Grands Lacs, International Alert en collaboration avec ONU FEMMES, $56 \mathrm{p}$.

14. WALTHER Olivier et KONE Moustapha, 2018, Commerce de réexportation des textiles et villes frontalières entre Niger et Nigéria. Les cahiers d'Outre-Mer, [en ligne], 278 juillet-décembre, ISSN 03735834, 465-491. 